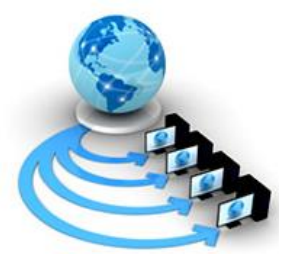

International Journal of Advanced Research in Computer Science

RESEARCH PAPER

\author{
Available Online at www.ijarcs.info
}

\title{
ANALYSIS OF IMAGE SEGMENTATION TECHNIQUES IN IMAGE PROCESSING
}

\author{
M.Praveena Kiruba bai \\ Associate Professor \& Research Scholar, \\ Department of Computer Science, \\ Lady Doak College, \\ Madurai-02.,India
}

\author{
Dr. G. Arumugam \\ Senior Professor and Head, \\ Department of Computer Science \\ School of Information Technology, \\ Madurai Kamaraj University,Madurai-21.
}

\begin{abstract}
Image segmentation plays a very important role for many image video and computer vision applications. It is quite a relevant research area due to its wide usage in the field of medical, remote sensing and image retrieval. Image segmentation is used to identifying the objects as well as boundaries in the images. Based on the image feature image segmentation clusters or classifies the image into different parts. There are several algorithms proposed for segmenting an image prior to its recognition. This paper highlights the strength and a limitation of classification techniques applied to texture classification and reviews various algorithms like active contour model, fuzzy $\mathrm{C}$ means, fuzzy $\mathrm{K}$ means algorithm etc used in the segmentation process.
\end{abstract}

Keywords: Segmentation, Classification, Compression, fuzzy C means, fuzzy K means

\section{INTRODUCTION}

Digital image processing processes the digital image with the help of computer algorithms. Image segmentation plays its significance among numerous activities. Segmenting an image is very important in the analysis of an image, representation of an object, processing different operations in the images and visualization. It is a technique that divides the image into different segments and helps in analyzing the segments for further information from the images. Various segmentation strategies are given below:

\section{A. Threshold-based segmentation:}

In this method, image pixels are divided with the help of intensity level of an image. This method is mainly used to distinguish the foreground objects from background images. The different techniques adopted for segmenting a digital image are slicing the image and Histogram thresholding.

In order to have multiple segments in an image, the Threshold-based segmentation method is extensible in using multiple thresholds. An algorithm that iteratively implements segmentation is the $K$-means clustering algorithm. It is mainly implemented when the segments are large enough and also it reduces the variances present during segmentation.

\section{B. Edge-based segmentation:}

This technique distinguishes the edges and identifies the boundaries in an object. It is a special approach used for segmenting the edges. The three important steps involved are to identify the edges from the given original image. The next step is to process those edges that have the object boundaries that are closed. Then transform the output to a segmented image by filling the boundaries of the object. The methods that are used to implement the concept of edges are mentioned below:
A digital image forms watershed due to 'flooding' because of its local minima and builds the 'dams' as the waterfronts collect together. As soon as the image is fully flooded, all the dams collectively form the watershed. This watershed that contains the edges is used for segmenting the images. The algorithm is used to trace out the waterlines in an image for separating the distinct regions.

In an image an active contour or snake technique is defined as a curve that modifies its position and contour until it best suits the predetermined conditions. It segments an image object by settling like a contracting snake that is held in the region of the boundary between the objects.

C. Region growing: The single pixels that are adjacent to each other are merged to a single segment. It makes use of a starting pixel called as the seed. This technique involves a selection process that identifies a seed from the given set. It examines all the four connected adjacent neighbours of the seed and merges the suitable neighbours. The neighbours that are merged, form the seed set. The initial seed is discarded from the seed set. The process of region growing stops when the seed set is empty.

D. Region-based segmentation: This method starts from the middle of an object. It then "grows" outward until it meets the boundaries of the object. It is opposite to the above- mentioned technique.

E. Texture measures: The texture characterizes coarseness or smoothness, directionality, intensity, color, and regularity or uniformity in an image.

Clustering techniques: In this method, similar patterns are grouped together based on some criteria. It is broadly divided into three categories: (1) Before segmentation process the original image (2) process the segmented result (3) adapt to the segmentation process. 
F. Agglomerative clustering This approach take apart every data point into separate clusters. It then proceeds to merge appropriate and relevant clusters into a single cluster. While merging, a dissimilarity matrix is used for analyzing which clusters are to be grouped together. The dissimilar clusters are grouped collectively that forms the smallest data entry point. After the merging gets completed, the next step is to choose a smallest entry point, by merging two new candidates.

\section{G. $K$-means clustering}

This algorithm is an iterative clustering algorithm that aims to find local maxima in every iteration.

\section{H. Artificial Neural Network}

A neural network is an artificial representation of human brain. It simulates the learning process. An artificial neural network is often called a neural network or simply neural net. The artificial neural network-based simulates the learning strategies of the human brain for the significance of decision making.

\section{LITERATURE REVIEW}

[1]. Constrained Active Contours for Boundary Refinement in Interactive Image Segmentation ${ }^{[1]}$ Nguyen Thi Nhat Anh, Jianfei Cai, Juyong Zhang and Jianmin Zheng.

The graph cut based algorithms and the region based algorithms that deals with Random Walks and Geodesic algorithms are sensitive to the user input in terms of either the number of seeds or the locations of seeds. Most of the state-of-the-art interactive image segmentation methods rely on additional user inputs either globally or locally refine the cutting contour. In terms of complex images, the user is often required to provide a lot of additional strokes or boundary points with the difficulty of laborious refinement or editing.

A constrained active contour method is proposed for boundary refinement combined with many existing interactive segmentation methods to improve their segmentation results without additional user inputs. This method is based on the convex active contour model which uses both the boundary and the regional information to find an "optimal" solution. A probability map is produced based on the hard segmentation map and the foreground/background Gaussian mixture models (GMM). The hard segmentation map indicates a value of 0 or 1 for the identification of background or foreground pixel respectively. They are used to represent the color distributions of the foreground and the background regions. With the probability map is calculated based on the foreground and background regions, pixel counter initialization was performed. The GMM probability map produced enhances the edge detection using the boundary term.

The proposed algorithm combined with Geodesic and random walks algorithms were tested with three images as input. The method was able to reduce the errors, smooth out the contours and provide geometric edges. It also generated stable results irrespective of different user inputs. The advantage is that the refinement method runs very fast due to the fact that the proposed constrained active contour model can be solved quickly by convex optimization.
[2]. Local- and Global-Statistics-Based Active Contour Model for Image Segmentation ${ }^{[2]}$ Boying $\mathrm{Wu}$ and Yunyun Yang

This paper discusses the drawbacks of different models thereby deriving a proposed model overcoming the limitations. The piecewise smooth models deal with images handling intensity inhomogeneity. It was most expensive. The region-scalable-fitting (RSF) model uses the local intensity information to overcome the difficulty caused by the intensity inhomogeneity but fails to deal with inhomogeneous images. The local and global intensityfitting (LGIF) energy model is derived from the collective significance of CV and RSF model. The local and the global information are taken into consideration in order to archive better segmentation results thereby defining a new energy functional. The model that is proposed differentiates the region that has similar intensity but with a different variance. The weight parameter $\mathrm{w}$ denotes the globalintensity fitting term and the value of this parameter is varied dynamically at diverse locations. The next step is to apply the split Bregman method. This method reduces the energy functional that is proposed in a proficient way.

The images that are taken into consideration are real and synthetic images. These images of the object have the same intensity means also considers the intensity variance. They have dissimilar variances for the object as well as for the background of the image. The results for another synthetic inhomogeneous image were discussed where the background and the two objects were corrupted by the severe intensity inhomogeneity. The result of the LGDF model and LGIF model cannot get the correct segmentation while the proposed method segments accurately.

The advantage of this approach is that it overcomes the difficulty caused by the intensity inhomogeneity.

[3]. Color image segmentation using histogram thresholding - Fuzzy C-means hybrid approach ${ }^{[3]}$ Khang SiangTan and NorAshidiMat Isa

Color is one of the most significant low-level features that can be used to extract homogeneous regions that are most of the time related to objects or part of objects. Fuzzy C-means (FCM) algorithm has been used extensively to improve the compactness of the regions due to its clustering validity and simplicity of implementation. It is a pixels clustering process of dividing pixels into clusters so that pixels in the same cluster are as similar as possible and those in different clusters are as dissimilar as possible. The limitation of this algorithm was it involves initialization difficulties of deciding the cluster number and obtaining the initial cluster centroids that are properly distributed. Recently, Ant colony algorithm proposed has high optimization but suffers from computational complexity. The proposed HTFCM consists of two modules, namely the histogram thresholding module and the FCM module. The histogram thresholding module is used for obtaining the FCM's initialization.

The global histograms thresholding has three phases. They are(1) peak finding technique (2) the region initialization (3) the merging process. The peak identifies 
the dominant peaks based on the intensity levels thereby eliminating the other peaks and the valleys. The next phase is to identify the dominating peak of each value and assign it to the nearest centroid until its value is less than the threshold. A merging algorithm merges these regions based on their color similarity. It is processed using the minimum Euclidean distance forming new cluster centres by reassigning the pixels to the nearest cluster centres. The merging also removes the number of clusters. The FCM algorithm partitions the image pixels into different clusters based on a criterion. The optimization of this algorithm is achieved by iterating to a small positive threshold. The quality of the cluster is calculated using Bezdek's partition coefficient and Xie and Beni function. The Bezdek's partition coefficient value should be larger and the Xie and Beni should be smaller.

Thirty images are selected to depict the performance of HTFCM out of which eight images are highlighted to specify the advantages of the proposed HTFCM approach, and the remaining 22 images are kept as supplementary images. The performance of the HTFCM approach gave a reasonable efficiency over the AS, the AFHA, and the IAFHA segmentation approaches. The advantage of this algorithm is it provides better performance and improved cluster quality than other approaches. It also avoids classification errors.

[4]. Segmentation of M-Fish Images for Improved Classification of Chromosomes with an Adaptive Fuzzy CMeans Clustering Algorithm ${ }^{[4]}$ Hongbao Cao and Yu-Ping Wang

The algorithm proposed called adaptive Fuzzy CMeans Clustering Algorithm segments and classifies Multicolor fluorescence in-situ hybridization (M-FISH) images. The chromosomes in this algorithm are highlighted with five different dyes. The DNA stain called DAPI attaches to the highlighted DNA considering each and every chromosome. Each dye has its own wavelength and their images were captured based on its wavelength visibility. The algorithm makes use of five different spectrums. It differentiates 24 different chromosome classes represented in the form of the human genome. For a normal cell the chromosomes are painted with the same color and for the abnormal cells, it has different colors indicating chromosomal abnormalities leading to genetic diseases and cancers.

Classification of Multicolor Fluorescence In Situ

Hybridization (M-FISH) Images With Sparse

Representation(Hongbao Cao et al, 2012)

Maximum-Likelihood Techniques for Joint Segmentation-

Classification of Multispectral Chromosome Images(Wade

C. Schwartzkopf 2005)

The proposed improved Adaptive Fuzzy Segmentation Algorithm uses the variance within a relatively larger neighbor area as the regulation term in the gain field. The first step is to initialize the variables $c_{k}$ and NC denoting the center of the clusters and the class no respectively. The second step is to upgrade the membership function and center of the clusters. The next step is to compute and upgrade the gain field. Stop the iteration when the greatest modified gain field and the greatest modified membership function is less than the tolerance value. The images are classified as foreground and background and the pixel of each class is labeled using the technique called as combinatorial labeling. The ratio of classification is calculated as the number of chromosome pixels that are classified accurately to the total number of pixels.

M-FISH database of 20 cells with 120 images (9 male, 11 female) was tested. The performance measure was calculated based on the false detection rate (FR) and correct detection rate (CR). The IAFCM shows advantages over AFCM, FCM and Otsu's methods with the lowest value of FR and provides the best ratio for classification. The results indicate that the IAFCM method has better segmentation performance than other methods. The drawback is it has not employed any pre-processing and post-processing steps.

[5]. Adaptive Fuzzy-K-means Clustering Algorithm for Image Segmentation ${ }^{[5]}$ Siti Noraini Sulaiman and Nor Ashidi Mat Isa

The need for the study is based on the disadvantages of the Fuzzy means algorithm. KM is although an exclusive clustering algorithm is dependent on initialization and is sensitive to outliers and skewed distributions. It converges to a local minimum and misses a small cluster. The Fuzzy C-means algorithm allows each data to belong to two or more clusters at a different degree of membership. The significant boundary between the elements is not clear if they do, or do not belong to a certain class. The moving K-means algorithm introduces a fitness concept together with the basic concept of KM to improve the segmentation performance. The anticipated AFKM is exclusively intended to include both the MKM and K-means algorithms. Every data must be allocated to the closest centre using the Euclidean distance. The recent location for every centre is considered

depending on the membership function. Every centres are ought to be assigned a specific value. The membership function is processed without the intrusion of outsiders. The degree of membership has its significance in establishing the new location of the centre. The degree to which the cluster belongs is analysed. The Higher degree of belongingness indicates a stronger relationship between the centre and its members and ensures a better data clustering. The new centre positions of the entire existing clusters are calculated based on the new (optimized) membership function. The performance analysis is carried out based on qualitative and quantitative analyses.

In this paper, ten out of those tested images were taken from indoor and outdoor electronics products. They were chosen to visualize the performance of the proposed algorithm. As the number of cluster sets increases the segmented images of the proposed AFKM are sharper and crispier with less noisy pixels. The proposed AFKM algorithm has outperformed other clustering algorithms. The clustering algorithm acquired a slightly higher processing time as compared to the conventional clustering MKM clustering algorithm.

[6]. Graph-based K-means clustering ${ }^{[6]}$ Laurent Galluccio a,c, Olivier Michel b, PierreComon a,n, and Alfredo.Herold 
The partitioning algorithm called the Prims trajectory necessitates the computation of a distance matrix between the data points, fails for a large volume of data. The Prim-based algorithm overcomes the difficulty of computing huge similarity matrices. The algorithm is based on a datadriven hierarchical classification applied to a variety of distance metrics. K-means algorithm is the simplest and most popular approach for solving clustering problems. The $\mathrm{K}$ means algorithm aims at finding a minimum dataset. Each data point is considered as the vertex in the graph and partitions the data set with a set of clusters. The first step is to construct an acyclic graph with a set of vertex and edges respectively. The total length of the tree is measured as the sum of all the edges. The minimum spanning tree was chosen based on the minimum length of all spanning trees. The construction of the MST acquires the knowledge of the dissimilarities between the pairs of vertices. An MST is built from two different Prim's trajectories. The next step is to set a threshold value. To initialize a threshold value the first step is to specify the spatial vertex distribution under the null hypothesis. The next step is to locate the probability of a subset of consecutive connections whose weights are specified below a given threshold. The third step is to link the probability of false alarm to the parameters. When the edges of some alarm are lesser than the threshold false alarm exists. To overcome the false alarm put right the minimal $\mathrm{k}$ value and calculate the threshold.

50 data samples are used to estimate the number of clusters present in the dataset. Apply the fixed parameters $\mathrm{k}$ and, $€$. It shows that the proposed method exhibits greater performance than other methods. The advantage of this Prim initialization method is that it produces significantly better clustering performance than the classical initialization methods.

[7]. Fuzzy C-Means Clustering With Local Information and Kernel Metric for Image Segmentation [7] Maoguo Gong, Yan Liang, Jiao Shi, Wenping Ma, and Jingjing Ma

This paper initially focuses on the drawbacks of the previously worked algorithm thereby suggesting the proposed method for segmentation. The conventional FCM algorithm fails to segment images corrupted by noise, outliers, and other imaging artifacts. FCM_S algorithm proposed, modified the objective function of FCM by introducing the spatial neighborhood term. It was very timeconsuming. Hence in FLICM, in order to improve the performance of restraining noise, the kernel distance measure was proposed. In recent years the kernel method is used in machine learning community. The kernel method transforms the complex nonlinear problems in original lowdimensional feature space to the transformed space. The FLICM introduces a trade-off weighted fuzzy factor and the kernel method. Initialize the cluster prototype and the array. The proposed algorithm has the following steps. The first step is to initialize the parameters and the random counter value. Set the loop counter to zero and the next step is to calculate the weighted factor and the distance measurement. Update the partition matrix and the cluster prototypes. Compare the vector value to the threshold. When the threshold value is maximum repeat the steps. Calculate the center of the local window. The distance variance is calculated using the kernel parameter. The performance metrics are measured for NNcut, FLICM and RFLICM are respectively affected by the noise to different extents.

Three synthetic test images are tested. The image with $128 \times 128$ pixels includes two classes with two intensity value taken as 20 and 120 . The other two images are constituted by $244 \times 244$ pixels and $256 \times 256$ pixels. The noise levels are 15\%, 20\% and 30\% corrupted by Gaussian noise and Salt \& Pepper noise with varying degrees, respectively. The SA(sum of total value) value of KWFLCM is larger than the other three compared algorithms in different noise levels (KWFLICM were up to $99.98 \%$, corrupted by $30 \%$ Salt \& Pepper noise). Experimental results show that the area segmented by the proposed method is clear and with high veracity.

The advantage of this algorithm is it improves the performance of image segmentation, as well as the robustness of the type of noise.

[8]. Automated two-dimensional K-means clustering algorithm for unsupervised image segmentation [8] Intan Aidha Yusoff, Nor Ashidi Mat Isa and Khairunnisa Hasikin.

The K-means algorithm makes use of the Euclidean distance to cluster the image. It clusters the image data using the value of the intensity of the pixel. The FCM algorithm permits the data to be a member. It clusters many data based on the different degrees of membership. The KM clustering is improvised by the moving $\mathrm{KM}$ clustering algorithm. It combines the fundamental concept of the novel algorithm with the concept of fitness.

The main objective of the novel algorithm is to construct an algorithm for the unsupervised data. On considering the noiseless image the optimum number is involuntarily determined based on the spatial and local domain. Unknowingly the number of clusters, this technique rules out the declaration of the clusters for the input of a noiseless image. The segmentation concept is to eliminate the irrelevant details that are present in the region ought to be segmented. These irrelevant details can be either denotes small regions, edges that are not segmented as individual regions. The proposed A2DKM algorithm uses the input digital image to be of 8-bit. It has 256 gray levels ranging the interval $[0,255]$. Let $p(x, y)$ denotes the location of the pixel at $\mathrm{x}$ and $\mathrm{y}$ coordinates with ' $\mathrm{p}$ ' as an intensity. Frequent studies have been made for the ease of the functioning of the clustering algorithm. This algorithm minimizes the loss of information and the pixels noise that interferes during segmentation. The intensity is frequently used and tailored in this algorithm. The algorithm distinguishes from KM based on the spatial and local information. The clusters are divided into four unlike quadrants. The net Euclidean distance is measured for every quadrant for all the members and it's compared. The process of splitting the clusters into quadrants and forming new clusters are repeated until the total Euclidean distance value is lesser than 0.5 . The very last number is initialized as the optimum or the final number of the cluster.

The qualitative analysis is implemented to prove the optimality of the number being processed. Three images are chosen for estimation. The images gave an enhanced result for the values $\mathrm{F}(\mathrm{I}), \mathrm{F}^{\prime} \mathrm{O}(\mathrm{I})$, and $\mathrm{Q}(\mathrm{I})$. If the value of the 
function reaches to a minimum value then it implicates that the results of segmentation produce a uniform, smooth and a homogeneous image.

The proposed algorithm was implemented using hundred gray-scale standard consumer images and they were processed using the conventional KM, FCM, and MKM clustering algorithms with the same number of clusters calculated using the A2DKM clustering algorithm. The Lake image is more homogeneous than the AFKM algorithm. The execution time is not better for the algorithm since it takes longer execution time. The qualitative and quantitative analyses provide better evidence than $\mathrm{KM}$, MKM, FCM and AFKM. The proposed algorithm complexity is analyzed using the execution time. The algorithm that is simple requires a shorter processing time, while a huge complex algorithm necessitates the longer time for execution.

[9]. Segmentation of Natural Images by Texture and Boundary Compression [9] Hossein Mobahi • Shankar R. Rao · Allen Y. Yang Shankar S. Sastry · Yi Ma

From the previous researches, it was analyzed that it is difficult to strike a good balance between objective measures that depend solely on the intrinsic statistics of imagery data. During the exploration of the objective measures the texture and their contours lack in their integrated representation.

This paper suggests a better segmentation result achieved based on the standard of image compression. It accurately counts the required bits that are needed to determine a natural image in terms of the texture and the boundaries. The proposed algorithm exactly determines the coding length for the texture features.Its mainly based on non-overlapping window numbers and the probability distribution of regions to be segmented. To adapt to various shapes and scales in an image, a ladder of numerous windows with its size is integrated. The minimum description length analyses the cluster for a mixed set of data. This algorithm is used for segmenting the images that are natural and it combines the novel clustering technique called as compression-based texture merging.

The first step is to construct Texture Features by defining the set of features $X$ considering its neighbourhood pixels and then stack the window as a column vector. Gaussian Mesh Markov Model (MMM) measures constantly the distribution of the pixels collectively in a window. In the distribution, the rate-distortion is calculated based on the optimal compression. It makes use of the covariance and the mean to calculate the textures that are to be encoded. To code an image from multiple windows know about the window of each region it resides. Use coding length to build the best compression algorithm.

During segmentation, the optimality is achieved through agglomerative approximation. To arrive at an accurate approximation, the image is implemented using a hierarchical and a novel function for coding length. The fineness of the segmentation is determined using the level of distortion. The $\varepsilon$ called as optimality best matches the perception of a human. For natural images the color spaces are compressed using the lossy compression technique.
The performance metrics of the TBES algorithm is based on four parameters PRI, VOI, BDI, and GFM. PRI and GFM with higher values yield better segmentation. VOI and BDE with minimum values yield segmentation better.CTM has not undergone a best estimate hence it does not justify a strong connection between image segmentation and its compression.

The database (Berkley Dataset) is divided into 200 training sets and 100 testing sets images. The segmentation is achieved using MATLAB. The run time average is estimated to be $164.59 \mathrm{~s}, 0.49 \mathrm{~s}$ and $412.51 \mathrm{~s}$ for construction features, map vicinity and segmentation respectively. The algorithm proposed has better performance. In the images few patterns might not agree with Gaussian texture. It has difficulty to initialise the super pixels of the lower level. The assessment of the texture regions are not stable and not in proper condition.

[10]. Non-cognitive Color and Texture Based Image Segmentation Amalgamation with Evidence Theory of crop images ${ }^{[10]}$ Masoom Jain and Mohammed G Vayada

Segmentation on trivial images is one of the difficult tasks in image processing. The scope of image processing has been evolved extensively in the field of Agriculture. This paper mainly focuses on crop images. The most important part in perceptual segmentation is feature extraction, color feature extraction, Texture feature extraction and Edge feature extraction etc. For retrieval and image indexing feature extraction is implemented. Feature extraction is a method of capturing the visual content of digital images for retrieval, indexing and for other different purposes. General and specific features are incorporated in this algorithm. Texture can be evaluated based on the characteristics like fine coarse, smooth, rippled, molded and irregular. One of the best tools for texture analysis of the image is GCLM. It defines NxN matrix and the size is equal to the largest gray level. The co-occurrence probability for pixels with gray levels is calculated. Then measure the degree of texture smoothness, contrast is used, and indicated as low when the image has constant gray levels. The Inverse difference gives the local homogeneity; it is high when a distribution of gray level is in limited range over the local images. To identify the color similarity between two images and to differentiate image based on their features the color moments are used. The Histogram is a bar graph that represents the tonal distribution in a digital image. Color Coherence vector is used to measure the spatial coherence of the pixels with a given color. It mixes the evidence from different sources of information to compute the probability of an event.

This work has been tested with more than 15 test images. Various parameters like PSNR, MSE, AD, MD, $\mathrm{NCC}$, and NAE are compared between color features, texture features, and the proposed method. The result was satisfactory in all the images with the very good PSNR value.

It helps to classify the image more clearly and accurately and it gives better and robust parameters values. It can be further modified to some extent based on the area of interest of images like in medical sector this theory can be proved efficient and research is open in particular field. 
III RESULTS AND DISCUSSION

The above -discussed algorithms were summarized and compared in the Tabular column given below.

\begin{tabular}{|c|c|c|c|c|c|}
\hline S.no & Authors & Title of the Paper & $\begin{array}{l}\text { Segmentation } \\
\text { Methods }\end{array}$ & Advantages & Limitations \\
\hline 1 & $\begin{array}{l}\text { Nguyen Thi Nhat } \\
\text { Anh }\end{array}$ & $\begin{array}{lr}\text { Constrained } & \text { Active } \\
\text { Contours } & \text { for } \\
\text { Boundary } & \\
\text { Refinement } & \text { in } \\
\text { Interactive } & \text { Image } \\
\text { Segmentation } & \\
\end{array}$ & $\begin{array}{l}\text { Convex Active } \\
\text { Contours } \\
\text { Method }\end{array}$ & $\begin{array}{l}\text { Very fast } \\
\text { Solves Quickly by } \\
\text { Convex } \\
\text { Optimization. }\end{array}$ & \\
\hline 2 & $\begin{array}{l}\text { Boying } \mathrm{Wu} \text { and } \\
\text { Yunyun Yang }\end{array}$ & $\begin{array}{l}\text { Local- and Global- } \\
\text { Statistics-Based } \\
\text { Active Contour } \\
\text { Model for Image } \\
\text { Segmentation }\end{array}$ & $\begin{array}{l}\text { Active Contours } \\
\text { Method- } \\
\text { standard } \\
\text { gradient descent } \\
\text { method }\end{array}$ & $\begin{array}{l}\text { It overcomes the } \\
\text { difficulty caused } \\
\text { by the intensity } \\
\text { inhomogeneity. }\end{array}$ & \\
\hline 3 & Khang SiangTan & $\begin{array}{lr}\text { Color } & \text { image } \\
\text { segmentation } & \text { using } \\
\text { histogram } & \\
\text { thresholding-Fuzzy } \\
\text { C-means } & \text { hybrid } \\
\text { approach } & \end{array}$ & $\begin{array}{l}\text { Histogram } \\
\text { thresholding- } \\
\text { Fuzzy C-means } \\
\text { hybrid approach }\end{array}$ & $\begin{array}{l}\text { It provides better } \\
\text { performance and } \\
\text { improved cluster } \\
\text { quality. } \\
\text { It avoids } \\
\text { classification } \\
\text { errors. }\end{array}$ & \\
\hline 4 & $\begin{array}{l}\text { Hongbao Cao and } \\
\text { Yu-Ping Wang }\end{array}$ & $\begin{array}{l}\text { Segmentation of } \\
\text { M-Fish Images for } \\
\text { Improved } \\
\text { Classification of } \\
\text { Chromosomes with } \\
\text { an Adaptive Fuzzy } \\
\text { C-Means Clustering } \\
\text { Algorithm }\end{array}$ & $\begin{array}{l}\text { Adaptive } \\
\text { Fuzzy C-Means } \\
\text { Clustering } \\
\text { Algorithm }\end{array}$ & $\begin{array}{l}\text { It has best } \\
\text { classification ratio }\end{array}$ & $\begin{array}{l}\text { The pre- } \\
\text { processing and } \\
\text { post-processing } \\
\text { steps are not } \\
\text { employed }\end{array}$ \\
\hline 5 & $\begin{array}{l}\text { Siti Noraini } \\
\text { Sulaiman and Nor } \\
\text { AshidiMat Isa }\end{array}$ & $\begin{array}{l}\text { Adaptive Fuzzy-K- } \\
\text { means Clustering } \\
\text { Algorithm for } \\
\text { Image } \\
\text { Segmentation }\end{array}$ & $\begin{array}{l}\text { Adaptive Fuzzy } \\
\text { K-means } \\
\text { Clustering } \\
\text { Algorithm }\end{array}$ & $\begin{array}{lr}\text { It } & \text { has } \\
\text { outperformed other } \\
\text { clustering } \\
\text { algorithms. } \\
\text { It has less noisy } \\
\text { pixels }\end{array}$ & $\begin{array}{l}\text { It has a slightly } \\
\text { higher } \\
\text { processing time }\end{array}$ \\
\hline 6 & $\begin{array}{l}\text { Laurent Galluccio, } \\
\text { Olivier Michel, } \\
\text { PierreComon and } \\
\text { Alfredo.Hero III }\end{array}$ & $\begin{array}{l}\text { Graph-based K- } \\
\text { means clustering }\end{array}$ & $\begin{array}{l}\text { The Prim-based } \\
\text { algorithm }\end{array}$ & $\begin{array}{l}\text { It has better cluster } \\
\text { performance }\end{array}$ & \\
\hline 7 & Maoguo Gong & $\begin{array}{l}\text { Fuzzy C-Means } \\
\text { Clustering } \quad \text { With } \\
\text { Local Information } \\
\text { and Kernel Metric } \\
\text { for Image } \\
\text { Segmentation }\end{array}$ & $\begin{array}{lr}\text { The } & \text { Kernel } \\
\text { Metric for } \\
\text { Image } \\
\text { Segmentation }\end{array}$ & $\begin{array}{l}\text { It improves the } \\
\text { performance of } \\
\text { image } \\
\text { segmentation. } \\
\text { The robustness of } \\
\text { the type of noise }\end{array}$ & \\
\hline 8 & Intan Aidha Yusoff & $\begin{array}{lr}\text { Automated two- } & \text { the } \\
\text { dimensional K- } \\
\text { means clustering } \\
\text { algorithm for } \\
\text { unsupervised image } \\
\text { segmentation }\end{array}$ & $\begin{array}{l}\text { Automated two- } \\
\text { dimensional K- } \\
\text { means } \\
\text { clustering } \\
\text { algorithm }\end{array}$ & $\begin{array}{l}\text { The clustering } \\
\text { algorithm has } \\
\text { relatively better } \\
\text { performance }\end{array}$ & $\begin{array}{l}\text { The complex } \\
\text { algorithm has a } \\
\text { longer } \\
\text { processing time. }\end{array}$ \\
\hline 9 & $\begin{array}{l}\text { Hossein Mobahi } \\
\text { Shankar R. Rao . }\end{array}$ & $\begin{array}{l}\text { Segmentation } \\
\text { Natural Images by }\end{array}$ & $\begin{array}{l}\text { Gaussian Mesh } \\
\text { Markov Model }\end{array}$ & $\begin{array}{l}\text { It improves the } \\
\text { performance of }\end{array}$ & $\begin{array}{l}\text { It has trouble to } \\
\text { properly form }\end{array}$ \\
\hline
\end{tabular}




\begin{tabular}{|l|l|l|l|l|l|}
\hline & & $\begin{array}{l}\text { Texture an } \\
\text { Boundary } \\
\text { Compression }\end{array}$ & TBES algorithm & $\begin{array}{l}\text { image } \\
\text { segmentation. }\end{array}$ & $\begin{array}{l}\text { low-level } \\
\text { superpixels used } \\
\text { as } \\
\text { initialization } \\
\text { It is unstable for } \\
\text { enough } \\
\text { large } \\
\text { windows size }\end{array}$ \\
\hline 10 & Masoom Jain & $\begin{array}{l}\text { Non-cognitive } \\
\text { Color and } \\
\text { Texture } \\
\text { Image } \\
\text { Segmentation } \\
\text { Amalgamation with } \\
\text { Evidence Theory of } \\
\text { crop images }\end{array}$ & $\begin{array}{l}\text { Grey level co- } \\
\text { occurrence } \\
\text { matrix }\end{array}$ & $\begin{array}{l}\text { It classifies image } \\
\text { more clearly and } \\
\text { accurately. } \\
\text { It gives better and } \\
\text { robust parameters } \\
\text { values. }\end{array}$ \\
\hline
\end{tabular}

\section{CONCLUSION}

In this paper, a partial survey of various image segmentation techniques has been accomplished. A comparative study is employed for various techniques. After evaluation of wellknown technique, it is clearly shown that the various methods can segment the image efficiently and provide accurate results. This work will be extended for a new algorithm for segmenting medical images which will provide more efficient result than the existing methods in near future. Computational time will also be considered to compare the techniques efficiently. Hence an elaborate methodology that high lights new vistas for developing more robust image segmentation technique is much sought.

\section{REFERENCES}

[1]. Nguyen Thi Nhat Anh, Jianfei Cai, Juyong Zhang and Jianmin Zheng,"Constrained Active Contours for Boundary Refinement in Interactive Image Segmentation, IEEE, 20 August 2012, Circuits and Systems (ISCAS), 2012 IEEE International Symposium,pp-870-873.

[2]. Boying Wu and Yunyun Yang, Local- and Global-StatisticsBased Active Contour Model for Image Segmentation Accepted 24 January 2012, Hindawi Publishing Corporation Mathematical Problems in Engineering Volume 2012, Article ID 791958,16 pages.

[3]. Khang SiangTan, NorAshidiMat Isa, Color image segmentation using histogram thresholding - Fuzzy Cmeans hybrid approach, Pattern Recognition 44 (2011),pp$1-15$.
[4]. Hongbao Cao and Yu-Ping Wang, Segmentation of M-Fish Images for Improved Classification of Chromosomes with an Adaptive Fuzzy C-Means Clustering Algorithm, IEEE, 2011,pp-1-8.

[5]. Siti Noraini Sulaiman and Nor AshidiMat Isa, Adaptive Fuzzy-K-means Clustering Algorithm for Image Segmentation, IEEE Transactions on Consumer Electronics ( Volume: 56, Issue: 4, November 2010 ), pp- 2661 - 2668.

[6]. Laurent Galluccio, Olivier Michel, PierreComon and Alfredo.Hero III Graph-based k-means clustering, Signal Processing Elsevier, 2012, 92 (9), pp-1970-1984.

[7]. Maoguo Gong, Member, IEEE, Yan Liang, Jiao Shi, Wenping $\mathrm{Ma}$, and Jingjing Ma, Fuzzy C-Means Clustering With Local Information and Kernel Metric for Image Segmentation, IEEE Transactions on Image Processing, VOL. 22, NO. 2, February 2013,pp- 573 - 584.

[8]. Intan Aidha Yusoff, Nor Ashidi Mat Isa, and Khairunnisa Hasikin, Automated two-dimensional K-means clustering algorithm for unsupervised image segmentation, Computers and Electrical Engineering, Volume 39 Issue3,April,2013, pp- 907-917.

[9]. Hossein Mobahi Shankar R. Rao · Allen Y. Yang · Shankar $\mathrm{S}$. Sastry Yi Ma, Segmentation of Natural Images by Texture and Boundary Compression, Computer Vision and Pattern Recognition, 18 Jun 2010,pp-86-98.

[10]. Masoom Jain, Mohammed G Vayada, Non-cognitive Color and Texture Based Image Segmentation Amalgamation with Evidence Theory of crop images, IEEE, International Conference on Sensing, Signal Processing and Security,2017,pp-160 - 165 . 